\title{
Frequency and inheritance of non-male producing clones in Daphnia magna: evolution towards sex specialization in a cyclical parthenogen?
}

\author{
Y. GALIMOV*, B. WALSER $\uparrow \& C . R$. HAAG $\dagger$ \\ *Institute of Developmental Biology, Russian Academy of Sciences, Moscow, Russia \\ $\dagger$ University of Fribourg, Ecology and Evolution, Fribourg, Switzerland
}

Keywords:

ecological genetics;

life history evolution;

sex-ratio.

\begin{abstract}
In Daphnia (Cladocera, Crustacea), parthenogenetic reproduction alternates with sexual reproduction. Individuals of both sexes that belong to the same parthenogenetic line are genetically identical, and their sex is determined by the environment. Previously, non-male producing (NMP) genotypes have been described in species of the Daphnia pulex group. Such genotypes can only persist through phases of sexual reproduction if they co-occur with normal (MP) genotypes that produce both males and females, and thus the breeding system polymorphism is similar to gynodioecy (coexistence of females with hermaphrodites), which is well known in plants. Here we show that the same breeding system polymorphism also occurs in Daphnia magna, a species that has diverged from $D$. pulex more than $100 \mathrm{MY}$ ago. Depending on the population, between $0 \%$ and $40 \%$ of $D$. magna females do not produce males when experimentally exposed to a concentration of the putative sex hormone methyl farnesoate that normally leads to male-only clutches. Natural broods of these NMP females never contained males, contrasting with high proportions of male offspring in MP females from the same populations. The results from a series of crossing experiments suggest that NMP is determined by a dominant allele at a single nuclear locus (or a several closely linked loci): NMP $\times$ MP crosses always yielded 50\% NMP and 50\% MP offspring, whereas MP $\times$ MP crosses always yielded $100 \%$ MP offspring. Based on cytochrome $c$ oxidase subunit I-sequences, we found that NMP genotypes from different populations belong to three highly divergent mitochondrial lineages, potentially representing three independent evolutionary origins of NMP in D. magna. Thus, the evolution of NMP genotypes in cyclical parthenogens may be more common than previously thought. Moreover, MP genotypes that coexist with NMP genotypes may have responded to the presence of the latter by partially specializing on male production. Hence, these populations of $D$. magna may be a model for an evolutionary transition from a purely environmental to a partially genetic sex determination system.
\end{abstract}

\section{Introduction}

Many species of plants show a sex allocation polymorphism, called gynodioecy, in which some individuals

Correspondence: Yan Galimov, Institute of Developmental Biology RAS, ul. Vavilova 26, 119334 , Moscow, Russia.

Tel.: +7 49913563 27; fax: +7 4991358012 ;

e-mail: yangalimov@gmail.com produce only female sexual organs, whereas other individuals are hermaphrodites, producing both female and male organs. Gynodioecy is believed to be an important intermediate step in the evolutionary transition from hermaphroditism to dioecy (separate sexes) in plants (Ross, 1970; Charlesworth \& Charlesworth, 1978; Charlesworth 2006). Research into the genetic basis of gynodioecy has shown that it can either be fully controlled by nuclear genes or by interactions between cytoplasmatic 
(usually mitochondrial) male suppressors and nuclear restorers. Most of the research has focused on cytonuclear interactions (e.g. Delph et al., 2007) because this appears to be the most common genetic basis of gynodioecy in plants. With cytonuclear interactions, the initial spread of the male suppressor can be explained by a selfish interest of cytoplasmatic elements because of their female-limited transmission. Yet, nuclear male-suppression mutants do not experience such a selfish spread, and full nuclear control thus implies an intrinsic fitness advantage of females at least when they are rare (when females become common, their advantage may be outweighed by negative frequency-dependent selection on sex ratios - a population consisting only of females could not persist). Hence, cases of nuclear control of gynodioecy may allow particularly interesting insights into the evolutionary advantages and disadvantages of gynodioecy.

In contrast to plants, gynodioecy appears to be rare in animals and its genetic basis is unknown (Schärer, 2009). A sex allocation polymorphism, which can be regarded as one form of gynodioecy, occurs in the water flea Daphnia pulex, a cyclical parthenogen, with environmental sex determination: Cyclical parthenogens reproduce clonally during most of the year. Yet, typically before the onset of harsh conditions such as freezing or drought, they produce males and subsequently undergo sexual reproduction during which diapause stages (called ephippia) are produced, which can survive the harsh conditions. Males, just as their clonal sisters, are produced clonally and are genetically identical to their mother. That is, clonal offspring can develop either into males or into females, depending on environmental cues, which are experienced by the mothers and are transmitted to the developing oocytes via hormones. Male production in Daphnia can also be experimentally induced by manipulating hormone levels in the growth medium (Olmstead \& Leblanc, 2002). Yet some clones of $D$. pulex have become unresponsive to the environmental cues that normally induce male production, and thus they never produce any males (Innes \& Dunbrack, 1993; Innes, 1997; Tessier \& Caceres, 2004). This can be regarded as a form of gynodioecy, because hermaphroditic clones, which produce both males and sexual females (here called male producing, MP clones), coexist with clones that only produce females (non-male producing, NMP clones). In contrast to typical gynodioecy, each individual is either a male or a female (separate sexes), and in Daphnia, the term 'gynodioecy' thus refers to the sexes of all members of a clone, not of an individual. Yet, just as in typical gynodioecy, NMP clones can only persist in populations if they co-occur with MP clones, at least in temporal populations, which obligately go through diapause. Populations with only NMP clones would go extinct under these circumstances because they would fail to produce diapause eggs, which require fertilization by males from MP clones.

The coexistence of MP and NMP clones within a population furthermore resembles gynodioecy because
NMP clones may have arisen because of male-suppression mutation. Indeed, in D. pulex, there is preliminary evidence suggesting that NMP may be under nuclear control (Innes \& Dunbrack, 1993). Hence, the evolution of NMP clones in D. pulex may represent an intermediate step between environmental and genetic sex determination, just as gynodioecy is intermediate between hermaphroditism and genetically determined dioecy in plants.

Extreme specialization of clones in male and female production has also been found in natural populations of the aphid Rhopalosiphum padi, another cyclical parthenogen (Rispe et al., 1999). Furthermore, some populations of Daphnia magna, a cyclical parthenogenetic congener of $D$. pulex, also show strong among-clone variation in sex allocation (Yampolsky, 1992) although it is yet unclear whether this is because of quantitative variation or because of the coexistence of MP and NMP clones. Finding NMP clones in D. magna would be highly interesting, because D. magna and D. pulex are highly divergent (> $100 \mathrm{MY}$, Colbourne \& Hebert, 1996), and gynodioecy would thus presumably have evolved independently in the two taxa. Together with the evidence for NMP clones in aphids, this may suggest that evolution of gynodioecy is common in cyclical parthenogens.

In the present study, we investigate the occurrence of NMP clones in D. magna using samples from eight populations and using the hormone methyl farnesoate, a highly efficient putative sex hormone of Daphnia, to test for clones that do not (even under raised hormone levels) produce males. Compared to the use of environmental cues for male induction, this design allows more efficient and more accurate classification into MP and NMP clones because given high enough hormone levels, MP clones will always produce male-only clutches during parthenogenetic reproduction (Olmstead \& Leblanc, 2002). Using mitochondrial markers, we also investigate the phylogenetic relationships among NMP clones from different populations and test for the inheritance of NMP using various crosses. Furthermore, we study the link between the temporal dynamics of sexual reproduction and the frequency of NMP clones in one particular population to gain insights into how NMP may be maintained in this population. Finally, we design a test for very rare males in NMP clones using mass cultures. This is important because the presence of such rare males has a strong effect on the predicted long-term evolutionary patterns of NMP. The results are discussed in the light of the potential origin and evolutionary maintenance of NMP clones in Daphnia.

\section{Materials and methods}

\section{Origin of samples}

Eight $D$. magna populations from geographically distant locations in Germany (one population) and Russia (seven 
populations) were studied: Ism: a eutrophic fish pond near Munich, Germany $48^{\circ} 12^{\prime} 57^{\prime \prime} \mathrm{N}, 11^{\circ} 40^{\prime} 42^{\prime \prime} \mathrm{E}$; SPb: a pond at former sludge fields in Saint-Petersburg $59^{\circ} 48^{\prime} 40^{\prime \prime} \mathrm{N}$, $30^{\circ} 07^{\prime} 59^{\prime \prime} \mathrm{E}$; MZ: a bird pond in Moscow Zoo $55^{\circ} 45^{\prime} 49^{\prime \prime} \mathrm{N}, 37^{\circ} 34^{\prime} 54^{\prime \prime} \mathrm{E}$; Syz: a former sludge pond in Syzran $53^{\circ} 12^{\prime} 46^{\prime \prime} \mathrm{N}, 48^{\circ} 29^{\prime} 15^{\prime \prime} \mathrm{E}$; $\mathrm{BN}$ : farm pond $50^{\circ} 09^{\prime} 26^{\prime \prime} \mathrm{N}, 43^{\circ} 23^{\prime} 31^{\prime \prime} \mathrm{E}$ about $200 \mathrm{~km}$ north of Volgograd; Vol: a pond in the Volgograd industrial area $48^{\circ} 31^{\prime} 48^{\prime \prime} \mathrm{N}, 44^{\circ} 29^{\prime} 13^{\prime \prime} \mathrm{E}$; Cav: a Daphnia cultivation pond at a sturgeon farm in the Volga delta $46^{\circ} 04^{\prime} 20^{\prime \prime} \mathrm{N}$, $47^{\circ} 43^{\prime} 45^{\prime \prime} \mathrm{E}$; Tam: a farm pond $45^{\circ} 13^{\prime} 18^{\prime \prime} \mathrm{N}, 36^{\circ} 48^{\prime} 30^{\prime \prime} \mathrm{E}$ near the north-eastern shore of the Black Sea. Clonal cultures were established using females isolated from plankton samples (populations Cav, SPb), females hatched from diapause eggs (populations BN, Vol, Ism) or both (populations Syz, MZ and Tam).

\section{Sampling procedures, establishment of clonal lines and line propagation}

Plankton samples were isolated using a plankton tow net. Samples were transported to the laboratory within $\leq 20 \mathrm{~h}$ after sampling, and clonal lines were established by isolating adult females from the samples, placing them individually into beakers and allowing them to produce clonal offspring. The clonal lines founded by these females were propagated in plastic beakers containing $70 \mathrm{~mL}$ of Daphnia medium (Klüttgen et al., 1994) and kept at room temperature and long photoperiods $(\sim 20 \mathrm{~h}$ of light). The cultures were fed daily with $0.5-1.0 \times 10^{6}$ Scenedsemus sp. cells per millilitre, and the Daphnia medium was exchanged two or three times a week.

Samples of ephippia (diapause eggs) were obtained from the uppermost $5 \mathrm{~cm}$ of pond sediments. Within 1 or 2 weeks after collection, sampled sediments were filtered through a series of sieves, and the $0.25-3-\mathrm{mm}$ fraction, which contains D. magna ephippia, was placed at $4{ }^{\circ} \mathrm{C}$ in the dark for at least 2 months. After this incubation period, ephippia were sorted out on ice and placed individually into cells of 96-cell immunological plates containing $0.2 \mathrm{~mL}$ Daphnia medium per cell. The plates were kept at $10^{\circ} \mathrm{C}$ and 13L: 11D photoperiod and checked daily. Juveniles that hatched from the ephippia were transferred individually to tubes containing $25 \mathrm{~mL}$ Daphnia medium and fed daily with $0.5-1.0 \times 10^{6}$ Scenedsemus sp. cells. Approximately 1 week after hatching from the ephippia, the hatchlings produced their first parthenogenetic clutches. At that moment, they were transferred to plastic beakers containing $70 \mathrm{~mL}$ of Daphnia medium and maintained as described above for the clonal lines established from plankton samples.

\section{Test for NMP clones: hormonal induction of male production}

To test for the presence and frequency of NMP clones in each population, we induced male production using the hormone methyl farnesoate. Hormone concentrations used in our study result in male-only clutches in MP clones in D. magna (Olmstead \& Leblanc, 2002; own unpublished). Thus, individuals that did not produce any male offspring under hormone exposure were classified as belonging to NMP clones. Adult females were isolated from the clonal cultures and placed individually into glass tubes containing $27 \mathrm{~mL}$ Daphnia medium and kept at $20{ }^{\circ} \mathrm{C}$ and a photoperiod of ca. $20: 4 \mathrm{~h} \mathrm{~L}: \mathrm{D}$ with $0.5-$ $1.0 \times 10^{6}$ Scenedsemus $\mathrm{sp}$. cells per $\mathrm{ml}$ per day as food. Every day, the females were moved to tubes with fresh medium, and at the same time they were checked for new broods, and tubes were checked for exuvia and newborn offspring. Once they had moulted (moult 1 in this experiment), females were transferred to medium containing methyl farnesoate at a concentration of $400 \mathrm{~nm}$, with all other conditions (temperature, photoperiod and food) remaining unchanged. Females were then kept in this hormone-containing medium (changed daily) until moult 2 when they were transferred back to hormone-free medium. In parthenogenetic Daphnia females, oocyte development starts after each moult, eggs are released into the brood pouch after the next moult, and juvenile Daphnia are released into the water during the third moult. In our experiment, females were thus exposed to the hormone during almost the entire period of ovary development, encompassing the entire period of hormone sensitivity (Olmstead \& Leblanc, 2002). The juveniles from the eggs released into the brood pouch just after moult 2 were counted and sexed under a stereo microscope once they were released to the water during moult 3. A total of 48 females (belonging to 48 clones from five populations) that were classified as NMP according to the hormone test were left in the hormone-containing medium also for a second, subsequent clutch (repetition of single females). In addition, in 42 cases (40 clones from five populations), we tested two or more females from the same clonal culture (repetition of different females from the same clone).

\section{Test of male production under outdoor conditions}

Clones failing to produce males in the hormone test may not be female-only clones under natural conditions, but may instead be insensitive to the hormone concentrations used in our test. To address this possibility, we assessed sex ratios of natural broods of individuals from the MZ population and tested their reaction to the hormone later (Methods described further below). In addition, we performed two further tests of male production in clones classified as NMP using outdoor cultures of NMP clones and outdoor control cultures of MP clones. First, we assessed late-season sex ratios in these cultures (late season is the time when males are most likely to be present). Second, because we could not exclude that males were present earlier in the season, we also tested for hatchlings from resting eggs in cultures 
that had been overwintered outside ('hatching test'). The presence of any hatchlings was considered as indication of sexual reproduction (and thus the presence of males) in the previous year. For both tests, we used cultures established in large buckets containing $60 \mathrm{~L}$ of Daphnia medium placed outside in the botanical garden of Fribourg. The cultures were eutrophicated using $\sim 100$ $\mathrm{g}$ of horse manure, and we added $500 \mathrm{~mL}$ of $50-\mu \mathrm{m}-$ filtered water from a natural, Daphnia-free pond as inoculum of natural food. The cultures were then grown without addition of further food, the buckets being covered with a fine mesh to prevent large debris from entering into the buckets, while allowing small debris and sunlight to penetrate. Population sizes in these cultures fluctuated, but were often in excess of 10000 individuals.

\section{Late-season sex ratios}

Sex ratios were assessed in ten cultures belonging to five NMP clones (two replicate cultures per clone) from populations BN, MZ (two clones), Vol and Ast. The latter population occurs in a pond near Astrakhan at $45^{\circ} 54^{\prime} 13^{\prime \prime} \mathrm{N}, 47^{\circ} 39^{\prime} 23^{\prime \prime} \mathrm{E}$ and is otherwise not included in this study. Sex ratios were also assessed in 11 cultures belonging to ten MP clones from populations $\mathrm{BN}$ (one clone), Ism (three clones), MZ (three clones), Vol (one clone) and Ast (two clones). All samples were taken between 11 November 2010 and 17 November 2010. The cultures had been grown outdoors for at least 4 months (since August 2010), whereas some of them had been grown outdoors since August 2008. Sex determination according to morphological characters was carried out using a stereo microscope.

\section{Hatching test}

The outdoor cultures were established in August 2008. In November, the volume was reduced to $\sim 10 \mathrm{~L}$, and the cultures were over-wintered outside in the dark until March 2009. During this period, the content of the containers froze solid, ensuring that no planktonic Daphnia survived. In March 2009, the containers were filled again to $60 \mathrm{~L}$ and checked for hatchlings twice a week by carefully sweeping a hand net repeatedly through the whole water column and removing any individuals caught. This test was carried out for three NMP clones (one from Ast and two from MZ) and for 16 MP clones (two from Ast, four from MZ, and ten from Ism), with two (clones from Ism) or three (all other clones) replicates in separate buckets for each clone. In replicates, in which more than five hatchlings were found, sexual reproduction was verified by assessing segregation of microsatellite loci that were heterozygous in the parent clone. For the microsatellite analyses, we extracted genomic DNA from single individuals using the HotSHOT protocol (Montero-Pau et al., 2008) and genotyped all individuals at 11 microsatellite loci (B. Jansen, S. Geldof, L. De Meester \& L. Orsini, unpublished data) that were heterozygous in the parent. We set up reactions of $10 \mu \mathrm{L}$, using $2 \times$ Type-it Multiplex PCR master mix (Qiagen, Venlo, The Netherlands). Cycling was performed following the recommendations of the manufacturer. Fragments were run on an ABI Prism 3130 Genetic Analyser, and fragment lengths were analysed using GeneMAPPER Software version 4.0 (Applied Biosystems, Foster City, CA, USA) with Gene Scan-500 LIZ as an internal size standard.

\section{Mitochondrial haplotypes of NMP clones}

To investigate the phylogenetic relationship among NMP clones from different populations, we sequenced a fragment of the mitochondrial COI gene. Genomic DNA was extracted from whole Daphnia females using Diatom DNA Prep kits (Isogen, Moscow, Russia). We amplified a 710-bp fragment of the cytochrome $c$ oxidase subunit I (COI) gene using primers LCOI490 and HCO2918 (Folmer et al., 1994). PCRs using the PCR kit from Isogen were performed according to standard protocols. The amplified fragments were purified and subsequently sequenced on an ABI PrIzm 3100 using BigDye v.l.1. Sequence analysis and alignment were performed using the LASERGene 6.0 software package (Burland, 2000). We obtained additional COI sequences of European D. magna (De Gelas \& De Meester, 2005) from Genbank and used these together with the fragments obtained in our study to build a haplotype network using the software tcs (Clement et al., 2000). This program estimates gene genealogies, based on absolute distances and parsimony, and is well suited for within-species data when divergences are low (Clement et al., 2000). The network was based on a final alignment of $609 \mathrm{bp}$. New sequences obtained in our study are deposited on Genbank (JF750768-JF750771).

\section{Population dynamics, sex ratios and sexual reproduction in the Moscow Zoo population}

To obtain data on the temporal dynamics of sex ratios, the prevalence of sexual reproduction and the frequency of NMP clones, we conducted a detailed study of the MZ population. This population occurs in a small $\left(1300 \mathrm{~m}^{2}\right)$, shallow ( $\sim 1 \mathrm{~m}$ deep) pond in the Zoo of Moscow (the Cormorant pond). The pond is covered by ice during the winter (approximately from November until March), with no planktonic D. magna population being present during this time. The planktonic population is re-established by hatching from ephippia.

The planktonic D. magna population of the MZ pond was sampled a total of 53 times in 2001, 2002 and 2006 throughout the growing season (i.e. the season when a planktonic population was present). Population densities (individuals per $\mathrm{m}^{3}$ ) were estimated from these samples (only 2002) by sampling $\sim 1 \mathrm{~m}^{3}$ of water and counting all D. magna individuals in the whole sample or in part of it. 
Because we observed spatial heterogeneity of $D$. magna densities within the pond, each density estimate was carried out at four different locations within the pond. In addition, 30-500 D. magna individuals were scored at each sampling date for sex and age class (adult vs. juvenile). Age classes were defined according to body size: the lower size-threshold for adults was defined as the size of the smallest females carrying broods or the smallest males with well-developed ventral chetae. To obtain an estimate of the prevalence of sexual reproduction, we distinguished between adult females carrying a parthenogenetic brood and adult females with an ephippial brood. Because, in D. magna, ephippia are exclusively produced by sexual reproduction, the relative frequency of females carrying ephippial vs. parthenogenetic broods is an estimate of the prevalence of sexual reproduction in the population. In addition, we noted the number of adult females carrying no brood and the clutch sizes of parthenogenetic broods.

To estimate not only adult sex ratios but also offspring sex ratios at birth, we isolated adult $D$. magna females from five plankton samples taken from the MZ pond, on 24 August 2006, 27 September 2006, on 11 September 2007, 2 October 2007 and on 28 August 2009. Thus, all samples were taken during a period, during which males are produced under natural conditions (see Results). Between 70 and 150 adult females were randomly selected and placed individually into $30 \mathrm{~mL}$ of Daphnia medium. They were fed $3 \times 10^{6}$ Scenedesmus sp. cells per day until they moulted for the first time. Offspring released at that moment were considered 'natural broods' because the number and sex of these offspring were already determined in nature (Ebert 8 Yampolsky, 1992; Olmstead \& Leblanc, 2002). The offspring were counted and sexed, and their mothers (including females that did not produce any offspring) were kept in clonal cultures and later tested for their reaction to methyl farnesoate. This allowed us to contrast the natural primary sex ratio between NMP and MP clones.

\section{Inheritance of NMP}

To investigate the inheritance of the NMP trait, we crossed females from NMP clones to males from MP clones and assessed NMP frequencies among the sexual offspring of these crosses. Two series of crosses were performed. First, seven NMP clones and eight MP clones established from hatchlings from ephippia collected from the MZ population in November 2005 were used to set up 16 crosses. For each cross, ten adult females (of the NMP clone) and five males (of the MP clone) were placed together into $30 \mathrm{~mL}$ of Daphnia medium. The males were either obtained directly from the clonal maintenance cultures or from the male-induction experiments. The tubes with the crosses were placed into short-day conditions (10L: 14D), which together with the high density (15 individuals in $30 \mathrm{~mL}$ of Daphnia medium) has been shown to stimulate sexual reproduction (De Meester \& De Jager, 1993). Any parthenogenetic offspring produced in these tubes were removed within 3 days, whereas the sexually produced ephippia were collected after 14 days, and incubated and hatched as described earlier. The Fl clones that hatched from the ephippia were scored as NMP or MP clones depending on their reaction to hormone exposure. The exact same procedure was also carried out for 19 control crosses, in which both parents were MP clones.

Second, because the first series of crosses resulted in only a limited number of Fl offspring from any particular cross, two further crosses were set up using larger numbers of males and females. The first of these crosses used again an MP and an NMP clone from the MZ population, whereas the second cross used an NMP clone from the MZ population and an MP clone from the Tam population. The experimental procedures were as in the first series of crosses (including removal of parthenogenetic offspring), with the exception that each cross was replicated four times, set up using 50 females and 25 males in $100 \mathrm{~mL}$ of Daphnia medium, and ephippia were collected after 4 weeks.

\section{Data analysis}

Confidence intervals for proportions were estimated with the GraphPad online calculator (http://www.graphpad. com/quickcalcs/ConfIntervall.cfm), using the modified Wald method (Agresti \& Coull, 1998), and differences in proportions between groups were tested with Fisher's exact tests or generalized linear models assuming a binomial error distribution using the statistical software JMP (SAS Institute, Inc, Cary, NC, USA). Throughout, two-tailed methods were used, except if observed proportions were zero or one (in these cases, true proportions can only deviate in one direction from the observed ones).

\section{Results}

\section{Occurrence and frequency of NMP clones in natural populations of Daphnia magna}

Daphnia magna clones from natural populations showed qualitative differences in sex composition of parthenogenetic broods that were laid after hormone exposure: Most of the clones produced only males (these clones were classified as MP clones), but a substantial proportion of clones produced only female broods (NMP clones). These results were $100 \%$ reproducible among different broods of single females or among different individual females from the same clone (48 repetitions for single females, 42 repetitions for different females of the same clone, see Materials and methods).

Non-male producing clones were found in the populations $\mathrm{BN}, \mathrm{MZ}, \mathrm{SPb}$, Tam and Vol, but not in the 


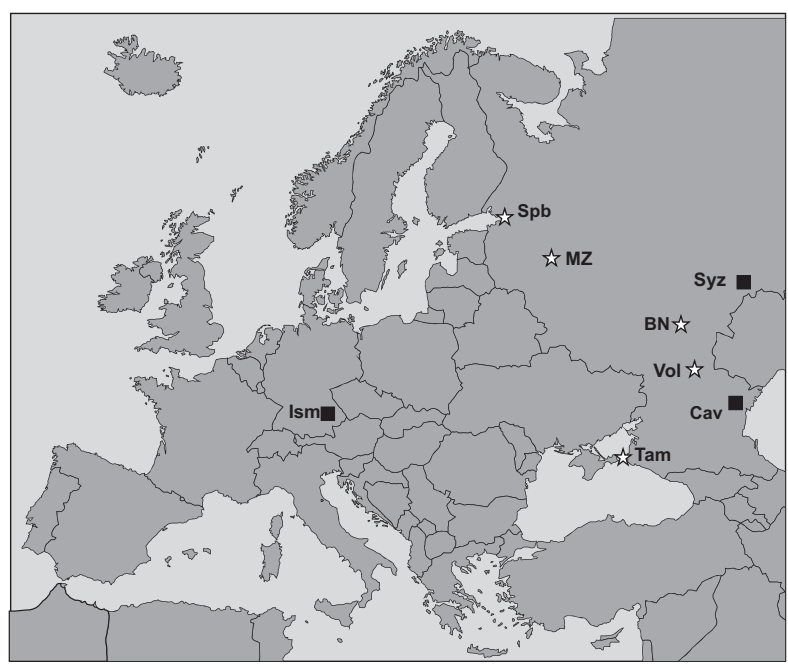

Fig. 1 Geographical location of the studied populations. Symbols indicate populations in which non-male producing (NMP) clones were found (stars) and populations in which no NMP clones were found (black squares).

populations Cav, Ism and Syz (Fig. 1, Table 1). We cannot exclude that NMP clones occur also in the latter three populations, but in our sample, the overall frequency of NMP clones in the latter three populations was significantly lower than in populations in which we found NMP clones (Fisher's exact test on pooled samples, $P<0.0001$, see also confidence intervals in Table 1). In samples of the ephippial (diapause) egg bank, the frequency of NMP clones (if present) ranged from 5\% to $21 \%$, with the differences among populations being significant (generalized linear model, $P=0.030$ ).
Frequencies of NMP clones (if present in the population) were higher in plankton samples (average weighted by sample size $=34 \%$, unweighted average $=32 \%$ ) than in ephippial samples (weighted average $=13 \%$, unweighted average $=14 \%$ ). The difference was highly significant if all plankton and all ephippial samples were pooled (Fisher's exact test, $P<0.001$ ) and also within the MZ population $(36 \%$ vs. $21 \%$, Fisher's exact test, all MZ plankton samples pooled, $P=0.016)$, but not within the Tam population (38\% vs. 19\%, Fisher's exact test, $P=0.36)$. Note, however, that only NMP frequencies among adult females could be estimated in the plankton samples (not frequencies in the entire population) because males and juveniles were not systematically surveyed. In the ephippia samples, NMP frequencies were estimated in the entire population because only females hatch from ephippia.

\section{Test of male production under outdoor conditions}

\section{Late-season sex ratios}

No males were detected among a total of 7840 individuals from outdoor mass cultures of five NMP clones originating from four different populations (between 487 and 3500 individuals were sexed per population). The $95 \%$ confidence limits for the proportion of males in the pooled sample are zero and 0.00056. In contrast, all of the MP cultures contained males, at estimated frequencies between $5 \%$ and $48 \%$ per population. Across the 11 MP cultures (belonging to 10 clones from five populations), we found 251 males and 653 females, which gives a overall sex ratio $28 \%$ males (with the $95 \%$ confidence limits being $25 \%$ and $30 \%$ ). If each culture was weighted equally, the average sex ratio in the MP cultures was $24 \%$ males.

Table 1 Numbers of male producing (MP) females and non-male producing (NMP) females in the population samples, as well as cytochrome $c$ oxidase subunit I (COI) haplotypes of MP and NMP clones (designation of already known haplotypes as in De Gelas \& De Meester, 2005). Proportion NMP designates the percentage of NMP among all tested females. The $95 \%$ confidence interval (CI) is given in brackets.

\begin{tabular}{|c|c|c|c|c|c|c|c|}
\hline \multirow[b]{2}{*}{ Population } & \multirow[b]{2}{*}{ Sample type } & \multirow[b]{2}{*}{ Sample date } & \multicolumn{2}{|c|}{ No. of females } & \multirow[b]{2}{*}{ Proportion NMP (Cl) } & \multicolumn{2}{|c|}{ COI haplotypes (cf. Fig. 2) } \\
\hline & & & $\mathrm{MP}$ & NMP & & MP & NMP \\
\hline \multirow[t]{6}{*}{$M Z$} & Ephippial & 7 November 2006 & 54 & 14 & $0.206(0.13-0.32)$ & $M Z$ & $M Z$ \\
\hline & Plankton & 24 August 2006 & 38 & 23 & $0.377(0.27-0.5)$ & & \\
\hline & Plankton & 27 September 2006 & 48 & 17 & $0.262(0.17-0.38)$ & & \\
\hline & Plankton & 11 September 2007 & 44 & 25 & $0.362(0.26-0.48)$ & & \\
\hline & Plankton & 2 October 2007 & 46 & 29 & $0.387(0.28-0.5)$ & & \\
\hline & Plankton & 28 August 2009 & 42 & 28 & $0.4(0.29-0.52)$ & & \\
\hline \multirow[t]{2}{*}{ Tam } & Ephippial & 16 July 2006 & 58 & 14 & $0.194(0.12-0.3)$ & H34, Tam & H34, Tam \\
\hline & Plankton & 16 July 2006 & 5 & 3 & $0.375(0.13-0.7)$ & & \\
\hline Ism & Ephippial & 1999 & 61 & 0 & $0(0-0.071)$ & $\mathrm{H} 34, \mathrm{H} 8, \mathrm{H} 12$ & \\
\hline $\mathrm{SPb}$ & Plankton & 12 September 2008 & 21 & 1 & $0.045(<0.001-0.24)$ & $\mathrm{H} 13$ & $\mathrm{H} 13$ \\
\hline $\mathrm{BN}$ & Ephippial & 12 June 2008 & 106 & 11 & $0.094(0.052-0.16)$ & $\mathrm{BN} 1, \mathrm{BN} 2$ & $\mathrm{BN} 1, \mathrm{BN} 2$ \\
\hline \multirow[t]{2}{*}{ Syz } & Ephippial & 30 April 2008 & 77 & 0 & $0(0-0.057)$ & $\mathrm{H} 8, \mathrm{MZ}$ & \\
\hline & Plankton & 30 April 2008 & 50 & 0 & $0(0-0.09)$ & & \\
\hline Vol & Ephippial & 16 June 2008 & 80 & 7 & $0.08(0.037-0.16)$ & H34 & $\mathrm{H} 34$ \\
\hline Cav & Plankton & 20 November 2008 & 80 & 0 & $0(0-0.055)$ & $\mathrm{H} 34, \mathrm{H} 18$ & \\
\hline
\end{tabular}




\section{Hatching test}

Despite the fact that no males were observed in the late-season samples, a low number of hatchlings was observed in several of the mass cultures of NMP clones that had been overwintered outdoors: One culture contained 46 hatchlings, the remaining cultures had either two hatchlings (one culture), one hatchling (three cultures) or zero hatchlings (four cultures). For each of the three clones, at least one hatchling was found, but also at least one of the cultures of each of the three clones contained no hatchlings. In contrast, $>200$ hatchlings were found in each of the 37 cultures of 16 MP clones from Ism, MZ and Ast. Most replicates ( 31 of 37) even had $>500$ hatchlings (we stopped counting at 500 hatchlings, but we estimated that some of the cultures had several thousands of hatchlings). Although this shows that number of hatchlings in NMP cultures was much lower than in MP cultures, these results still suggest that sexual reproduction and thus male production had occurred in the monoclonal cultures of NMP clones. The microsatellite genotypes (Table S3) of the 46 offspring from the NMP culture with the largest number of hatchlings confirmed segregation of alleles just as in normal sexual reproduction: The exact same alleles were present in the offspring and the parent at all 11 loci. Because no other clone in our cultures had exactly the same microsatellite profile as this parent clone, contamination can be excluded as a possible explanation of these results. Furthermore, we found heterozygotes and each of the two homozygotes at each locus in the offspring, with genotype ratios closely conforming to the expected Mendelian ratios $25 \%$ of each of the two homozygotes and $50 \%$ of heterozygotes). This shows that normal segregation had taken place during reproduction, thus suggesting normal sexual reproduction, although automictic parthenogenesis cannot be excluded. Normal sexual reproduction was also verified in all 37 cultures of MP clones, and not a single case of contamination was detected.

\section{COI haplotypes of MP and NMP clones}

In the eight studied populations, we found a total of nine distinct haplotypes of the 609-bp COI fragment (Table 1, Fig. 2). The analysis of the haplotype network (Fig. 2) showed that within each population, NMP-clones and MP clones had the same or closely related COI haplotypes, but NMP clones (as well as MP clones) from different populations had, in some cases, highly divergent haplotypes: In our data, NMP occurred in three different parts of the network, separated by eight to 11 mutational steps.

\section{Population dynamics and sexual reproduction in the MZ population}

The density of the MZ population in 2002 (Fig. 3) showed two peaks, one in late spring and one in mid-summer. Estimates of the total population size of the pond are somewhat hampered by the spatial heterogeneity of density within the pond. However, the available data (Fig. 3) point to the presence of 100-200 hatchlings per $\mathrm{m}^{3}$ or $1.5-3 \times 10^{5}$ for the whole pond. Because each hatchling is a product of sexual reproduction, this is also an estimate of the number of genetically unique clones present in the population. These then reproduced clonally during the season and reached census population sizes of the order of $10^{8}$ during the peak in early June, whereas the population size during the July 'bottleneck' was still in the order of $10^{6}$ individuals. The combined data from all years also show that although a few males were already produced in May/June, the vast majority of male production occurred in mid-August to October (Fig. 4a), and that a sharp switch occurred during the month of September from exclusively parthenogenetic reproduction of females in the early season to almost $100 \%$ sexual reproduction (production diapause eggs) in the late season (Fig. 4b).

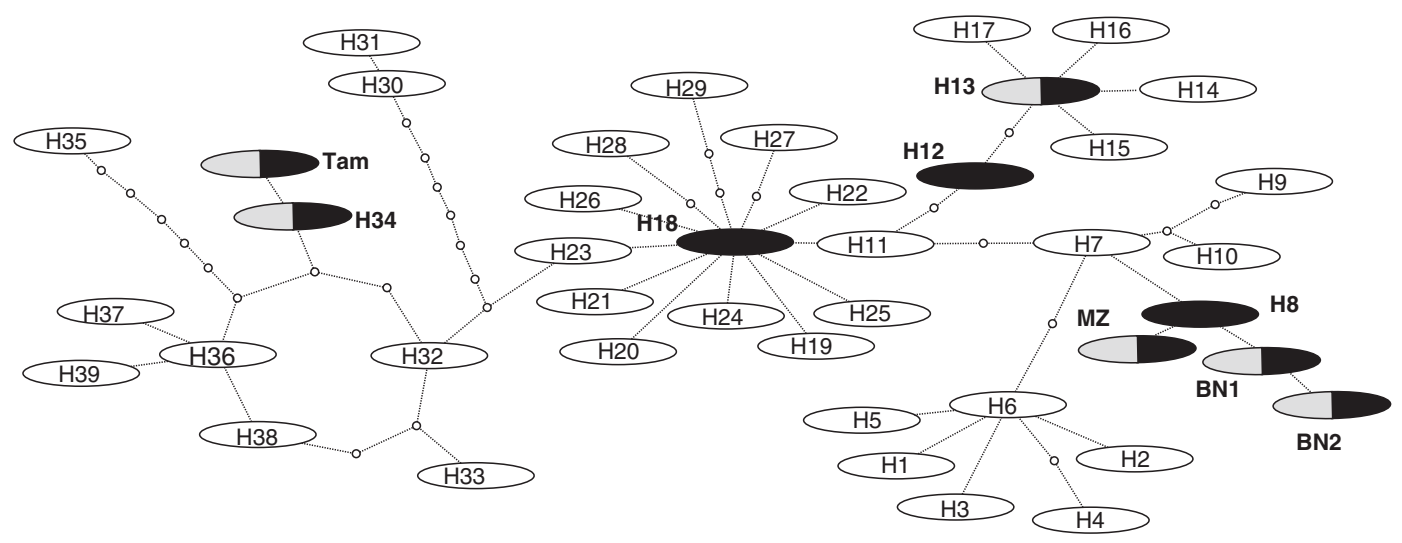

Fig. 2 Cytochrome $c$ oxidase subunit I haplotype network of Daphnia magna showing the haplotypes found in male producing clones (black) and in non-male producing clones (grey) in the current study. The other haplotypes were retrieved from Genbank. The designation of haplotype names follows De Gelas \& De Meester (2005), except for formerly unknown haplotypes (BN1, BN2, MZ and Tam). 


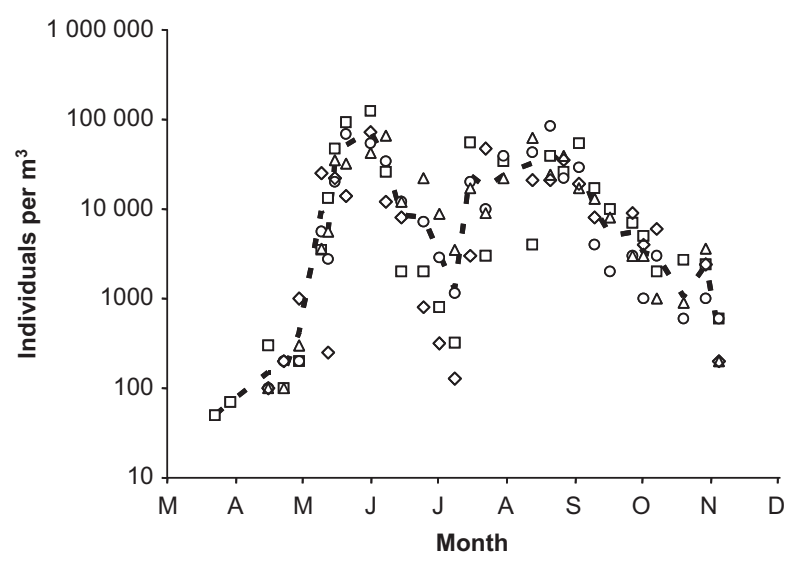

Fig. 3 Population size estimates for the MZ population in 2002: Densities at four different locations within the pond (symbols) and their means (dashed line) for each sampling date.

\section{Natural sex ratios of clutches produced by MP and NMP clones}

When investigating the natural broods of individuals later identified (by the hormone test) as belonging to MP or NMP clones (Table 2), there was no significant difference between NMP clones and MP clones in the proportion of individuals that had no broods (Fisher's exact tests for each sample independently, all $P>0.05$ ). However, the natural broods of NMP clones never contained males, whereas the average proportion of male broods in MP clones was $89 \%$. This difference was highly significant in all three samples where it could be tested (Fisher's exact tests, all $P \leq 0.007)$. Note that these samples were taken during periods when the MZ population was known to produce males (Fig. 4a). In addition, in the two samples, where ephippial and nonephippial broods were observed, ephippial broods tended to occur more often in NMP clones (proportion of ephippial broods among all nonempty broods, Fisher's exact tests, $P=0.078$ and $P=0.083$ for the samples of 24 August 2006 and 27 September 2006, respectively). The clutch sizes of parthenogenetic broods were very similar and not significantly different between NMP and MP clones ( $t$-tests within each sample, all $P>0.05$, Table 2 ).

\section{Inheritance of NMP}

The first series of crosses yielded a total of $85 \mathrm{Fl}$ clones from the $16 \mathrm{NMP} \times \mathrm{MP}$ crosses and $57 \mathrm{Fl}$ clones from the $19 \mathrm{MP} \times \mathrm{MP}$ control crosses (Table S1). None of the offspring of the control crosses were NMP $(95 \%$ confidence limits for the proportion of NMP offspring in $\mathrm{MP} \times \mathrm{MP}$ crosses $=0-0.054)$. In contrast, 41 of the 85
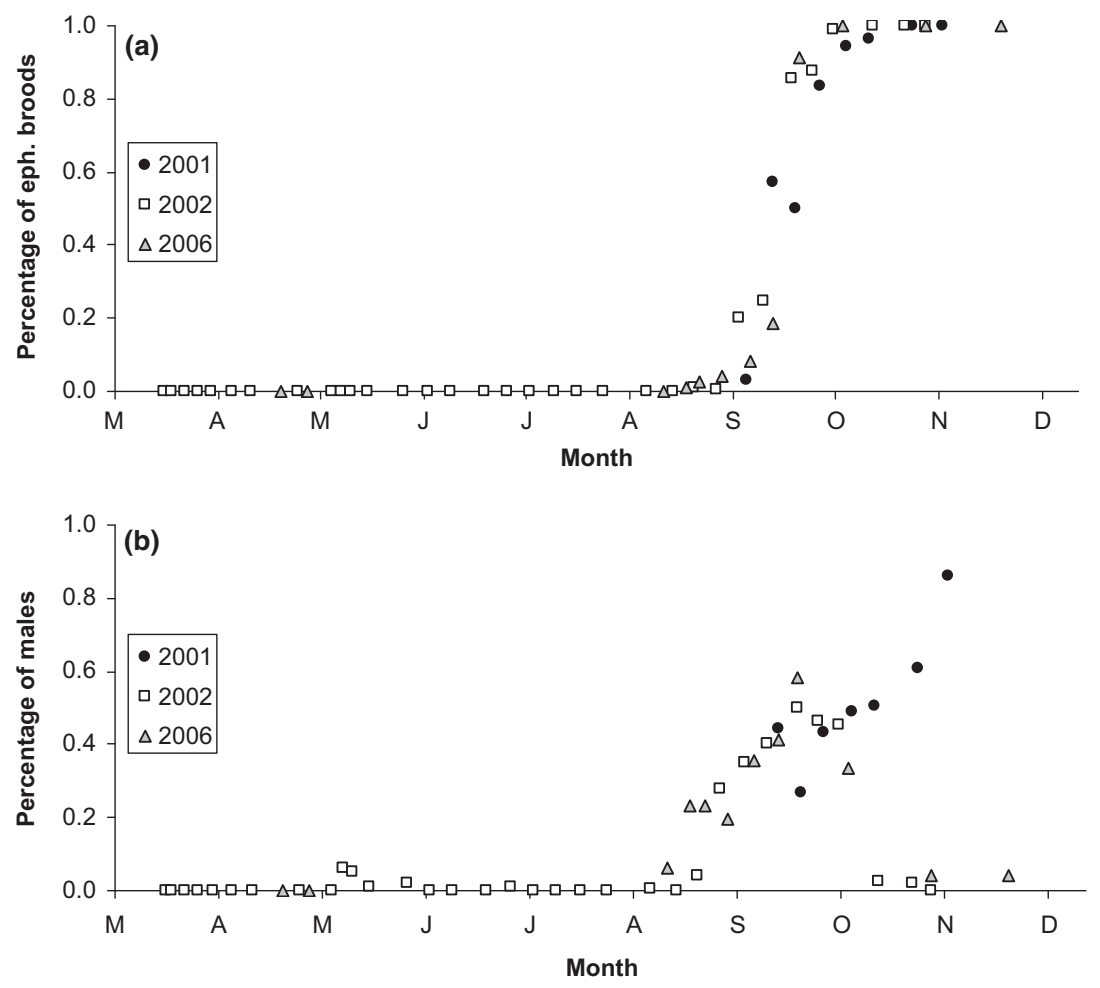

Fig. 4 (a) Prevalence of sexual reproduction (percentage of ephippial broods among nonempty broods) and (b) sex ratio (percentage of males) in plankton samples from the MZ population in 2001, 2002 and 2006. 
Table 2 Natural broods of male producing (MP) and non-male producing (NMP) clones (MZ population). No mixed broods were observed.

\begin{tabular}{|c|c|c|c|c|c|c|c|}
\hline \multirow[b]{3}{*}{ Sampling date } & \multirow[b]{3}{*}{ Hormone reaction } & \multicolumn{4}{|c|}{ Natural broods } & \multirow[b]{3}{*}{$\mathrm{SR}^{*}$} & \multirow[b]{3}{*}{$\mathrm{BS} \dagger(\mathrm{SD})$} \\
\hline & & \multicolumn{2}{|c|}{ Parth. } & \multirow[b]{2}{*}{ Eph. } & \multirow[b]{2}{*}{ No brood } & & \\
\hline & & $q$ & $\hat{0}$ & & & & \\
\hline \multirow[t]{2}{*}{24 August 2006} & NMP & 7 & 0 & 3 & 15 & 0 & $1.4(0.23)$ \\
\hline & MP & 2 & 10 & 0 & 26 & 0.83 & $1.7(0.23)$ \\
\hline \multirow[t]{2}{*}{27 September 2006} & NMP & 0 & 0 & 14 & 3 & NA & NA \\
\hline & MP & 1 & 6 & 25 & 16 & 0.86 & $2.0(0.2)$ \\
\hline \multirow[t]{2}{*}{11 September 2007} & NMP & 21 & 0 & 0 & 4 & 0 & $2.0(0,2)$ \\
\hline & MP & 2 & 33 & 0 & 9 & 0.94 & $2.3(0.9)$ \\
\hline \multirow[t]{2}{*}{28 August 2009} & NMP & 12 & 0 & 0 & 16 & 0 & $2.3(1.5)$ \\
\hline & $\mathrm{MP}$ & 4 & 24 & 0 & 14 & 0.86 & $2.5(0.8)$ \\
\hline
\end{tabular}

*SR Offspring sex ratio (number of male offspring divided by the total number of offspring) in the 'natural' parthenogenetic broods. †BS Clutch size of parthenogenetic broods (zero broods excluded) and standard deviation (SD).

NA not assessed.

offspring of the NMP $\times$ MP crosses were NMP. The difference between the two types of crosses was highly significant (Fisher's exact test on all NMP $\times$ MP and all $\mathrm{MP} \times \mathrm{MP}$ crosses pooled, $P<0.001$ ), and the proportion of NMP among the offspring from the NMP $\times$ MP crosses did not significantly differ from 0.5 (average $=0.48$, binomial test on all NMP $\times$ MP crosses pooled, $P=0.83$, $95 \%$ confidence limits $=0.379-0.587)$. There was not a single NMP $\times$ MP cross, in which this proportion differed significantly from 0.5 (binomial tests, all $P \geq 0.25$ ), although sample sizes (and thus the power) for individual crosses was small (Table S1).

The objective of the second series of crosses was to test the hypothesis of $50 \%$ inheritance of the NMP trait within single $\mathrm{NMP} \times \mathrm{MP}$ crosses (thus, no $\mathrm{MP} \times \mathrm{MP}$ control crosses were carried out). The results indicated a close fit to this hypothesis (Table S2): First, 58 (exactly $50 \%$ ) of $116 \mathrm{Fl}$ offspring clones from the MZ (NMP) $\times$ Tam (MP) cross were NMP $(95 \%$ confidence limits $=$ $0.410-0.590)$. Second, $27(47 \%)$ of $58 \mathrm{Fl}$ offspring clones from the cross between the NMP and MP clones from MZ were NMP (binomial test for a difference from $50 \%, P=0.69,95 \%$ confidence limits $=0.343-0.592$ ). Combining all NMP $\times$ MP crosses from both series of crosses, the observed proportion of NMP clones among the Fl offspring was $48.6 \% \quad(N=259)$, with a $95 \%$ confidence interval for the true proportion of $42.6-$ $54.7 \%$.

\section{Discussion}

\section{Occurrence of NMP clones in Daphnia magna}

Our study presents clear evidence for the existence of non-male producing genotypes in $D$. magna. Not a single male was produced by NMP clones in our hormoneexposure experiments, and also none of the natural broods of these genotypes contained any males, even though investigation of natural broods took place at a time when males were produced in the population. Furthermore, no males were observed in a large sample of individuals from outdoor cultures of NMP clones at a time when control MP cultures contained a high proportion of males. Despite this, the results from our mass cultures suggest that the NMP phenotype might not be $100 \%$ complete. Rather, NMP clones may rarely still produce males, although we cannot exclude the alternative explanation of rare resting ephippia production by automictic parthenogenesis. Rare males have also been found in asexual species, including obligately asexual strains of D. pulex (Innes \& Hebert, 1988; Innes et al., 2000). If they do indeed exist in NMP clones, their evolutionary maintenance is a puzzle, because if males are only very rarely produced, deleterious mutations affecting male-limited genes should be nearly neutral, and may thus accumulate, which should lead to a complete halt of male production. A possible solution of this puzzle is that NMP clones in D. magna might be rather young on an evolutionary time scale. Regardless of the question of how such rare males are maintained, the question of whether or not they exist has important consequences for the evolutionary dynamics of NMP clones. These will be discussed together with potential origin of NMP clones below.

\section{Inheritance of NMP in Daphnia magna}

The proportion of NMP clones among Fl offspring of $\mathrm{NMP} \times \mathrm{MP}$ crosses was very close and not significantly different from 0.5, whereas MP $\times$ MP crosses never produced NMP offspring. Similar data were obtained in D. pulex (Innes \& Dunbrack, 1993) although with a somewhat higher uncertainty, likely due to misclassification of some MP clones as NMP because male production was studied using environmental cues rather than a hormone treatment (Olmstead \& Leblanc, 2002). The results of the crossing experiments are best explained by NMP being determined by a dominant allele at a single 
nuclear locus (or several closely linked loci), because this would lead to both the observed $1: 1$ segregation ratios in all NMP $\times$ MP crosses and the observed absence of NMP offspring in all MP $\times$ MP crosses. Because NMP $\times$ NMP crosses are impossible (at least if the NMP phenotype is complete), this model would suggest that all NMP clones are heterozygous $\mathrm{Mm}$ at the locus conferring NMP, whereas MP clones are homozygous for the recessive allele $(\mathrm{mm})$. In contrast, if NMP was recessive, $\mathrm{NMP} \times \mathrm{MP}$ crosses should consistently produce $50 \%$ NMP offspring only if all MP clones were heterozygous, but in that case MP $\times$ MP crosses should produce $25 \%$ NMP offspring, which was not the case.

Potential alternative explanations for the results of our crosses may include maternally inherited selfish elements, such as parasitic bacteria (e.g. Wolbachia), or selfish genetic elements in maternally inherited organelles or on sex chromosomes (Merçot et al., 1995). Because these selfish elements can occur together with suppressor mutations, various segregation ratios, including $1: 1$ ratios, may be observed (Van Damme et al., 2004; Bailey \& McCauley, 2005). In Daphnia, where both sexes are believed to be genetically identical, it is unlikely that selfish genetic elements on sex chromosomes exist. There is also no evidence for Wolbachia or other parasites that induce a strong female-biased sex ratio (Fitzsimmons \& Innes, 2005; D. Ebert, unpublished). In addition, we treated ten NMP females of the MZ and Vol populations with tetracycline, and this treatment did not recover their ability to produce males (data not shown). Our results could, however, be explained by an interaction between a selfish mitochondrial gene causing NMP and a nuclear male-restorer gene with a recessive restorer allele, as observed in some gynodioecious plants (Barr, 2004). Under this scenario, NMP-conferring mitochondrial genotypes are fixed within populations (if they are not fixed, some $\mathrm{MP} \times \mathrm{MP}$ crosses would result in NMP offspring), but restorer-loci are polymorphic with all MP clones being homozygous for the restorer allele and all NMP clones being heterozygous (NMP homozygotes for the nonrestorer allele do not occur because they would require crosses between two NMP clones). However, studies of such systems in plants have shown that interpopulation crosses often result in a breakdown of the restoration (and thus to a high proportion of malesterile individuals), because within populations, NMP alleles are matched by restorer alleles that are specific to each population (Barr, 2004). We performed one such interpopulation cross between the distant Tam and MZ populations (with highly divergent mitochondrial haplotypes, Fig. 2), but offspring of this cross still produced NMP clones with a 1: 1 segregation ratio. This result could only be explained by an interaction between cytoplasmic selfish elements and nuclear restorers, if these restorers are universal in their action between phylogenetically distant mitochondrial haplotypes. Thus, although a simple nuclear control with a dominant allele conferring NMP appears to be the most likely explanation for our results, we cannot entirely exclude a more complicated genetic mechanism. Interestingly, in another breeding system polymorphism in Daphnia, the obligate asexuality of some strains of $D$. pulex, first data also indicated a simple nuclear control with a single dominant allele conferring obligate asexuality (Innes $\delta$ Hebert, 1988). However, recent results suggest that several loci are involved (Lynch et al., 2008).

\section{Origin of NMP clones}

We found NMP clones in three divergent mitochondrial haplotype clades of $D$. magna. Under the assumption that NMP is indeed determined by a dominant allele at a single, nuclear locus, the nuclear NMP allele always gets cotransmitted with its mitochondrial haplotype. Therefore, the existence of NMP in three haplotype clades that are separated by eight to 11 mutational steps suggests one of the following possibilities: (i) NMP is old and has occurred (or still occurs) also in intermediate haplotypes (i.e. the mitochondrial haplotypes associated with it have diverged by mutation). (ii) NMP is transmitted from one mitochondrial background to another via rare males. (iii) NMP has multiple, independent origins. Further studies are needed to distinguish between the three alternatives.

\section{Evolutionary maintenance of NMP clones}

Under the assumption that NMP is indeed determined by a dominant allele at a single, nuclear locus, NMP frequency would be reduced by half every sexual generation (a two-fold 'transmission disadvantage') because only half of the offspring of NMP $\times$ MP crosses are NMP, whereas all offspring from MP $\times$ MP crosses are MP (and NMP $\times$ NMP matings do not occur or are very rare). Hence, NMP clones should, on average, produce at least twice as many ephippia as MP clones (per original hatchling in the beginning of the season) for NMP to be maintained in the populations (Lloyd, 1975; Charlesworth \& Charlesworth, 1978). In Daphnia, this could be achieved, if the NMP clones have a sufficient advantage during either clonal reproduction or ephippia production (female sexual reproduction), for instance because they do not need to produce males. It has been shown in rotifers that selection favours lower investment in sexual reproduction during the parthenogenetic phase (Carmona et al., 2009). Indeed, we observed a higher proportion of NMP clones in planktonic samples than in ephippial samples, suggesting that the frequency of NMP clones increased during or after hatching from the ephippia. In addition, NMP clones tended to have a higher proportion of ephippial natural broods than MP clones. However, it is yet unknown whether these effects are strong enough to compensate for the two-fold transmission disadvantage during sexual reproduction and thus to lead to stable maintenance of NMP in these populations. 
If NMP clones have a sufficient advantage to be maintained, a stable polymorphism would be expected because NMP is unlikely to go to fixation because of additional costs owing to negative frequency-dependent selection in favour of MP when NMP becomes common (in the absence of rare NMP males, pure NMP populations would be deemed to go extinct because of failure to reproduce sexually).

Although our data suggest an advantage of NMP during clonal reproduction and perhaps also during female sexual reproduction, the reasons for these advantages are unknown. The most widely used explanation for a coexistence of hermaphrodites and females (gynodioecy) in plants (when it is not driven by selfish genetic elements) is inbreeding depression. In contrast to hermaphrodites, females are unable to self-fertilize, and thus, if selffertilization occurs frequently enough and the costs of it are strong enough, this process may explain the maintenance of females. Similar arguments have been used to explain NMP/MP coexistence in North American D. pulex (Innes \& Dunbrack, 1993). This work suggests that the equilibrium frequency $P_{n}^{*}$ of NMP clones is given by

$$
P_{n}^{*}=(1-2 X F) /(2-2 X F),
$$

where $F$ is the proportion of sexually reproducing individuals in MP clones that are females and $X$ is the survivorship of offspring of MP $\times$ MP matings relative to survivorship of offspring of NMP $\times$ MP matings (Innes $\delta$ Dunbrack, 1993). The latter is believed to be $<1$ because of inbreeding depression. Indeed, inbreeding depression is well known in Daphnia (Innes, 1989; De Meester \& Vanoverbeke, 1999; Haag et al., 2002), but rates of inbreeding (within-clone mating is genetically equivalent to self-fertilization) in large populations of Daphnia are likely to be low because of high numbers of different clones and no assortative mating (Mitchell et al., 1995; De Meester \& Vanoverbeke, 1999). Our conservative estimate of the number of unique clones hatching each spring in the MZ population is $1.5 \times 10^{5}$. Even with strong clonal selection during the season, the probability of within-clone mating in the MZ population thus hardly exceeds $10^{-4}$. Moreover, this population was shown to be genetically very diverse (Yampolsky $\&$ Kalabushkin, 1992; Yampolsky \& Galimov, 2005; B. Walser \& C. R. Haag, unpublished). Based on these estimates, $X$ should not be $<0.9995$.

Assuming that the frequency of $20.6 \%$ of NMP in the ephippia sample of the MZ population represents an equilibrium value of NMP and assuming that inbreeding is rare (so that $X$ is close to 1 ), the expected proportion of females among the sexually reproducing individuals (males and sexual females) of the MP clones is $F=0.37$. This suggests that MP clones may specialize to some degree on their male function (i.e. they may produce more males than they would in the absence of NMP clones), as predicted by the model of Innes \& Dunbrack (1993). However, the value of $F$ in populations consisting only of
MP clones is unknown, and the parameter $F$ is difficult to estimate in Daphnia populations, because some females may never reproduce sexually. For a parthenogenetic female, producing female offspring is both investment into future parthenogenetic reproduction and future sexual reproduction, whereas producing male offspring is only investment in future sexual reproduction via the male function. Therefore, it can be speculated that sex ratios among sexually reproducing individuals are, if anything, female-biased (i.e. $F \geq 0.5$ ) in populations that consist of MP clones only. This suggests that the low expected value of $F$ in the MZ population together with the high parthenogenetic investment in males of MP clones (Table 2) might be indications of a certain degree of male specialization of the MP clones in MZ.

\section{Conclusion}

Our study shows that the breeding system polymorphism in D. magna parallels that in the highly divergent D. pulex as well as in aphids (Innes \& Dunbrack, 1993; Rispe et al., 1999), thus suggesting that the evolution of NMP clones in cyclical parthenogens may not be uncommon. Moreover, our data hint at partial male specialization in MP clones that coexist with NMP clones, thus suggesting that not only male suppressor genotypes but also partial female suppressor genotypes may have evolved. In purely sexual organisms, the predicted outcome would be a clear distinction into genotypes that only produce males and genotypes that only produce females (Innes $\delta$ Dunbrack, 1993). One potential reason why this appears not to happen in Daphnia is that females are needed for parthenogenetic reproduction, even in MP clones (Innes \& Dunbrack, 1993).

\section{Acknowledgments}

We are grateful to L. Yu. Yampolsky and N.S. Mugue for contributing materials and for helpful advice and discussions throughout the project. We thank V.R. Alekseev and D. Ebert for contributing samples, C. Haag-Liautard, A.A. Minin, and E.F. Uryuppova for advice and comments, and Moscow Zoo and N. I. Skuratov for sampling permits and support. We are grateful to two anonymous reviewers for their comments that helped to improve the manuscript. This work has been supported by the Swiss National Science Foundation and the Russian Foundation of Basic Research.

\section{References}

Agresti, A. \& Coull, B.A. 1998. Approximate is better than "exact" in interval estimation of binomial proportions. Am. Stat. 52: 119-126.

Bailey, M.F. \& McCauley, D.E. 2005. Offspring sex ratio under inbreeding and outbreeding in a gynodioecious plant. Evolution 59: 287-295. 
Barr, C.M. 2004. Hybridization and regional sex ratios in Nemophila menziesii. J. Evol. Biol. 17: 786-794.

Burland, T.G. 2000. DNASTAR's Lasergene sequence analysis software. Methods Mol. Biol. 132: 71-91.

Carmona, M.J., Dimas-Flores, N., García-Roger, E.M. \& Serra, M. 2009. Selection of low investment in sex in a cyclically parthenogenetic rotifer. J. Evol. Biol. 22: 1975-1983.

Charlesworth, D. 2006. Evolution of plant breeding system. Curr. Biol. 16: R726-R735.

Charlesworth, B. \& Charlesworth, D. 1978. A model of evolution of dioecy and gynodioecy. Am. Nat. 112: 975-997.

Clement, M., Posada, D. \& Crandall, K. 2000. TCS: a computer program to estimate gene genealogies. Mol. Ecol. 9: 1657-1660.

Colbourne, J.K. \& Hebert, P.D.N. 1996. The systematics of North American Daphnia (Crustacea: Anomopoda): a molecular phylogenetic approach. Phil. Trans. R. Soc. Lond. B 351: 349360.

De Gelas, K. \& De Meester, L. 2005. Phylogeography of Daphnia magna in Europe. Mol. Ecol. 14: 753-764.

De Meester, L. \& De Jager, H. 1993. Hatching of Daphnia sexual eggs: 1. Intraspecific differences in the hatching responses of D. magna eggs. Freshw. Biol. 30: 219-226.

De Meester, L. \& Vanoverbeke, J. 1999. An uncoupling of male and sexual egg production leads to reduced inbreeding in the cyclical parthenogen Daphnia. Proc. R. Soc. B 266: 2471-2477.

Delph, L.F., Touzet, P. \& Bailey, M.F. 2007. Merging theory and mechanism in studies of gynodioecy. Trends Ecol. Evol. 2: $17-24$.

Ebert, D. \& Yampolsky, L.Y. 1992. Family planning in Daphnia: when is clutch size determined? Russ. J. Aquat. Ecol. 1: 143147.

Fitzsimmons, J.M. \& Innes, D.J. 2005. No evidence of Wolbachia among Great Lakes area populations of Daphnia pulex (Crustacea: Cladocera). J. Plankton Res. 27: 121-124.

Folmer, O., Black, M., Hoeh, W., Lutz, R. \& Vrijenhoek, R. 1994. DNA primers for amplification of mitochondrial cytochrome $c$ oxidase subunit I from diverse metazoan invertebrates. Mol. Mar. Biol. Biotech. 3: 294-299.

Haag, C.R., Hottinger, J., Riek, M. \& Ebert, D. 2002. Strong inbreeding depression in a Daphnia metapopulation. Evolution 56: 518-526.

Innes, D.J. 1989. Genetics of Daphnia obtusa: genetic load and linkage analysis in a cyclical parthenogen. J. Heredity 80: 6-10.

Innes, D.J. 1997. Sexual reproduction of Daphnia pulex in a temporary habitat. Oecologia 111: 53-60.

Innes, D.J. \& Dunbrack, R.L. 1993. Sex allocation variation in Daphnia pulex. J. Evol. Biol. 6: 559-575.

Innes, D.J. \& Hebert, P.D.N. 1988. The origin and genetic basis of obligate parthenogenesis in Daphnia pulex. Evolution 42: 10241035.

Innes, D.J., Fox, C.J. \& Winsor, G.L. 2000. Avoiding the cost of males in obligately asexual Daphnia pulex (Leydig). Proc. R. Soc. Lond. B 267: 991-997.

Klüttgen, B., Dülmer, U., Engels, M. \& Ratte, H.T. 1994. ADaM, an artificial freshwater for the culture of zooplankton. Water Res. 28: 743-746.

Lloyd, D.G. 1975. The maintenance of gynodioecy and androdioecy in angiosperms. Genetica 45: 325-339.

Lynch, M., Seyfert, A., Eads, B. \& Williams, E. 2008. Localization of the genetic determinants of meiosis suppression in Daphnia pulex. Genetics 180: 317-327.
Merçot, H., Atlan, A., Jacques, M. \& Montchamp-Moreau, C. 1995. Sex-ratio distortion in Drosophila simulans: co-occurence of a meiotic drive and a suppressor of drive. J. Evol. Biol. 8: 283-300.

Mitchell, S., De Meester, L., Carvalho, G. \& Weider, L.J. 1995. No evidence for kin-preferential swarming in Daphnia. J. Anim. Ecol. 64: 777-779.

Montero-Pau, J., Gómez, A. \& Muñoz, J. 2008. Application of an inexpensive and high-throughput genomic DNA extraction method for the molecular ecology of zooplankton diapausing eggs. Limn. Oceanogr.: Methods 6: 218-222.

Olmstead, A.W. \& Leblanc, G.A. 2002. Juvenoid hormone methyl farnesoate is a sex determinant in the crustacean Daphnia magna. J. Exp. Zool. 293: 736-739.

Rispe, C., Bonhomme, J. \& Simon, J.C. 1999. Extreme life-cycle and sex ratio variation among sexually produced clones of the aphid Rhopalosiphum padi (Homoptera: Aphididae). Oikos 86: 254-264.

Ross, M.D. 1970. Evolution of dioecy from gynodioecy. Evolution 24: $827-828$.

Schärer, L. 2009. Tests of sex allocation theory in simultaneously hermaphroditic animals. Evolution 63: 1377-1405.

Tessier, A.J. \& Caceres, C.E. 2004. Differentiation in sex investment by clones and populations of Daphnia. Ecol. Lett. 7: 695-703.

Van Damme, J.M.M., Hundscheid, M.P.J., Ivanovic, S. \& Koelewijn, H.P. 2004. Multiple CMS-restorer gene polymorphism in gynodioecious Plantago coronopus. Heredity 93: 175181.

Yampolsky, L.Yu. 1992. Genetic variation in the sexual reproduction rate within a population of a cyclic parthenogen, Daphnia magna. Evolution 46: 833-837.

Yampolsky, L.Y. \& Galimov, Y.R. 2005. Evolutionary genetics of aging in Daphnia. Zhurn. Obsch. Biol. 66: 416-424.

Yampolsky, L.Y. \& Kalabushkin, B.A. 1992. Genetical variability in an intermittent population of Daphnia magna. Genetika 28: $41-50$.

\section{Supporting information}

Additional Supporting Information may be found in the online version of this article:

Table S1 Inheritance of non-male producing in the first series of crosses.

Table S2 Inheritance of non-male producing in the second series of crosses.

Table S3 Segregation of alleles at 11 microsatellite loci in the $46 \mathrm{Fl}$ offspring in one of the replicate culture of MZ01-04. 